\title{
Biomass and Molecular Characteristics of Multi-tillering Miscanthus Mutants
}

\author{
Geung-Joo Lee ${ }^{1 *}$, Lili Zhang ${ }^{2}$, Young In Choi ${ }^{1}$, Sung Jin Chung ${ }^{1}$, \\ Yong Kweon $\mathrm{Yoo}^{2}$, Dong Sub Kim ${ }^{3}$ and Sang Hoon Kim ${ }^{3}$ \\ ${ }^{1}$ Department of Horticulture, Chungnam National University, Daejeon 305-764, Korea \\ ${ }^{2}$ Department of Horticultural Science, Mokpo National University, Jeonnam 534-729, Korea \\ ${ }^{3}$ Radiation Breeding and Genetics, Korea Atomic Energy Research Institute, Jeongup 580-185, Korea
}

\begin{abstract}
Compared to wide ranges of genetic variation of natural populations, very limited Miscanthus cultivar has been released. This study was the first report on the development of Miscanthus cultivar by means of radiation breeding. Seeds of M. sinensis were initially exposed to gamma rays of $250 \mathrm{~Gy}$ for $24 \mathrm{~h}$, generated from a ${ }^{60} \mathrm{Co}$ gamma-irradiator. The irradiated seeds were sown and then the highly tiller-producing mutants were selected for this study. Biomass-related parameters including tiller number, plant height, stem diameter, and leaf number were measured. Ploidy level and internal transcribed spacer (ITS) were investigated to characterize the mutants compared to wild type (WT) Miscanthus. Plant height and tiller number were negatively related, where multi-tillering mutants were relatively short after 4 month growth. However stem diameter and leaf number were greater in mutants. All the materials used in this study were diploid, implying that the mutants with greater tiller numbers and stem diameter were not likely related to polyploidization. Based on the sequence of ITS regions, the mutants demonstrated base changes from the gamma irradiation where $\mathrm{G}+\mathrm{C}$ content (\%) was decreased in the ITS1, but increased in ITS2 when compared to WT sequence. ITS2 region was more variable than in ITS1 in the mutants, which collectively allows identification of the mutants from WT. Those mutants having enhanced tillers and allelic variations might be used as breeding materials for enhanced biomass-producing Miscanthus cultivars.
\end{abstract}

Key words - Internal transcribed spacer (ITS), Plant height, Ploidy level, Stem diameter, Tiller number

\section{Introduction}

The carbon dioxide released from various industrial processes has been increased at a rate of approximately $3 \%$ yearly for the past 50 years. Heaton et al. (2003) indicated that this increase would be continued by combustion of fossil fuels, which caused the significant change in climate. Research reports indicated increasing interest in the use of plant material as a direct source of bioenergy and biomaterials (Henry 2010). Renewable energy consumption rose by $6 \%$ to over 8 quadrillion BTU (British Thermal Unit) between 2009 and 2010, and the biomass share of the renewable consumption increased from 49 to $53 \%$ during the years of 2006 to 2010 (Eynon, 2011). This fact demonstrates that the biomass plays an important role in the renewable energy resources.

The genus Miscanthus belongs to the family Poaceae and

${ }^{\star}$ Corresponding author. E-mail : gjlee@ $@$ cnu.ac.kr most of the species were originated from tropical and subtropical or temperate regions of Asia (Greef and Deuter, 1993). Out of more than 20 species, only M. sinensis, M. sacchariflorus, M. floridulus, and M. x giganteus (hybrid between $M$. sinensis and M. sacchariflorus) are of interest for biomass production or industrial uses (Jones and Walsh, 2001; Sorensen et al., 2008). Three major components of Miscanthus lignocellulosic feedstock include cellulose, hemicelluloses, and lignin, which range $43-52 \%, 25-33 \%$, and $9-13 \%$, respectively, depending on species and genotypes (Brosse et al., 2012). Holocellulose content (cellulose+hemicellulose) was generally higher in M. sinensis (78 82\%) than M. sacchariflorus (76 $78 \%$ ) or M. x giganteus (75 77\%) (Brosse et al., 2012).

Most of the reports on the genus Miscanthus attributed to agronomic or physiological studies including yield trials, rhizome propagation, and resource use efficiency (Beale and Long, 1997; Venendaal et al., 1997; Lewandowski et al., 2000; Pyter et al., 2010). Some of taxonomics and phylogenetics 
provided basic biology for the plant (Greef and Deuter, 1993; Hodkinson et al., 2002), and recent molecular studies by using genomics information accelerated development of molecular markers for gene mapping from the complex genome (Rayburn et al., 2009; Swaminathan et al., 2012).

Physical mutagens like gamma ray or fast neutron were reported to induce phenotype change in many species, which was mostly related to base change for point mutation, sequence shift by deletion or insertion of some DNA bases, or even chromosome aberrations (Tindall et al., 1988; Li et al., 2002; Dhanavel et al., 2012). The induced mutants are partly considered in a breeding program for improving some characters including yield, quality, earliness, resistance to pathogens, and tolerance to abiotic stresses (van Harten, 1998). Ribosomal genes consisted of $18 \mathrm{~S}, 5.8 \mathrm{~S}$, and $28 \mathrm{~S}$ were reported to be conserved in the closely related species, while internal trans- cribed spacers (ITS) between the conserved regions were found to be highly variable, allowing the tool used as taxonomic marker (Chen et al., 2010).

The objective of this study was to evaluate the potential use of the ionizing mutagenesis via a gamma radiation for induction of biomass-enhanced Miscanthus mutants. We first compared biomass-related traits between wild type (WT) Miscanthus and its mutants, and then characterized the mutants by investigating molecular changes at the DNA and ploidy levels.

\section{Materials and Methods}

\section{Mutant induction using a gamma radiation}

Seeds of diploid Miscanthus sinensis were collected from an isolated Imja island of Jeonnam province in 2010, and then
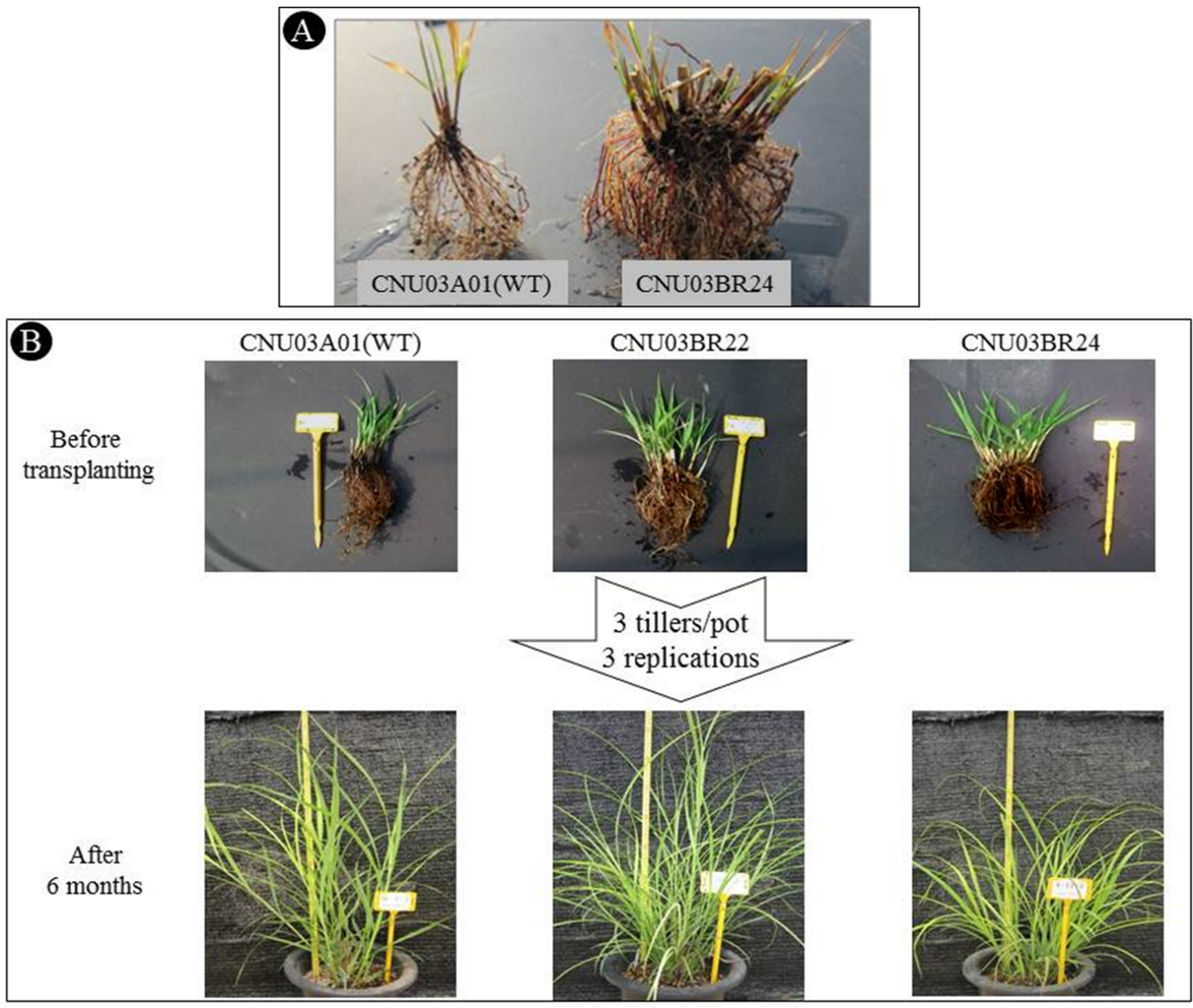

Fig. 1. One seed-originated plants and initial selection of higher tillering mutant (A) and division of the genotypes into 3 tillered plant in a pot (B) for a conformational experiment in a consecutive year. 
were exposed to $\gamma$-rays of 100, 125, and $150 \mathrm{~Gy}$ for $24 \mathrm{hrs}$ as generated by a ${ }^{60} \mathrm{Co}$ gamma-irradiator (150 TBq capacity; ACEL, Canada) at the Korea Atomic Energy Research Institute, Jeongeup. All treated seeds (M1) were then directly sown in a plastic greenhouse, and the M2 plants were grown in a successive season to select mutants. The germinated plants which showed strong seedling growth were transplanted into an individual pot. Finally two greater tillering mutants exposed to 125 Gy were selected and used in this study (Fig. 1).

\section{Cultivation and growth measurement}

In a consecutive year, the selected mutants and WT (no treatment) which were originally grown from one seed which was treated with 125 Gy gamma radiation were divided into three-tillered plants per pot with three replications for measurements of biomass-related traits (Fig. 1). The divided multi- tillering mutants and WT were investigated monthly from June 1st to October 16th, 2011. The plant growth parameters including plant height, tiller number, stem diameter and leaf number were measured. The plant height was recorded as the perpendicular distance from the soil at plant's base to the highest point reached with all the parts in their natural position. The stem diameter was measured at $5 \mathrm{~cm}$-height from soil surface.

\section{Measurement of nuclear DNA content}

The ploidy level of WT (diploid), M. sacchariflorus (tetraploid) as a reference, and two mutants was examined by using a flow cytometry (Partec, Germany) equipped in the Institute of Agricultural Science and Technology, Naju city, Jeonnam province, Korea. About $1 \mathrm{~cm}^{2}$ segments of fresh leaf tissue was chopped in $500 \mathrm{ul}$ of the nucleus isolation buffer in a petri
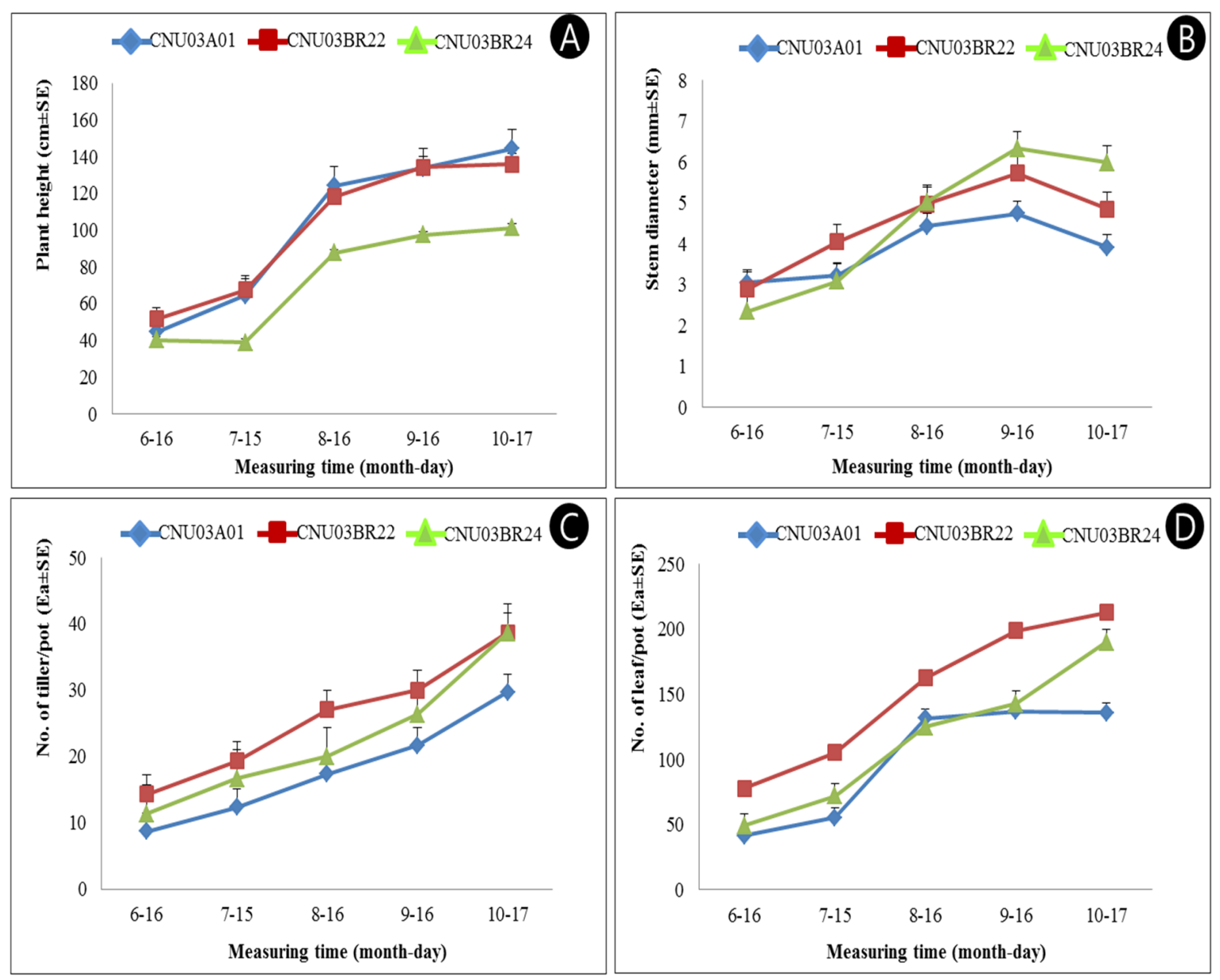

Fig. 2. Comparative growth of WT and two mutants based on plant height (A), stem diameter (B), number of tiller (C), and number of leaf (D) by growth stages. 
dish. The extracted leaf nuclei were stained in $2 \mathrm{ml}$ of CyStain ${ }^{\circledR}$ UV Precise P250 containing 4',6'- diamidino-2phenylindole (DAPI). The resultant solution was filtered through $30 \mu \mathrm{m}$ nylon mesh into a $5 \mathrm{ml}$ collection tube. The relative fluorescence of total DNA of single nuclei was analyzed and recorded. Prior to measuring the nuclear DNA content, the flow cytometry was calibrated with a chicken red blood cell to adjust all the parameters including gain value, flow speed, and an internal standard.

\section{ITS analysis for identifying mutants}

To determine the sequence of a $5.8 \mathrm{~S}$ ribosomal gene and flanking ITS regions, another grass belonging to a Poaceae family, zoysiagrass (Zoysia japonica) ITS (NCBI genebank no. HQ600481) sequence was used to amplify the Miscanthus ITS region. The forward (ATTGTCGTGACCCTGACCAAAAC) and reverse primer (TGGGGTCGTGGTCCAAGCAATG) sets were designed and the amplicons were successfully obtained and visualized on $1 \%$ agarose gel (Fig. 4 , top left), and then sequenced directly.

\section{Data analysis}

Data collected from growth measurements were analyzed for statistical significance by the SPSS software, version 19. Four growth parameters in three replications were recorded monthly and data were compared among the genotypes (WT and two mutants), which were plotted with a standard error on the graph (Fig. 2).

\section{Results and Discussion}

After exposing of Miscanthus seeds to gamma ray at 125 Gy, two variants showing higher tillering were selected (Fig. $1 \mathrm{~A})$, which were subjected to performance evaluation in the following year (Fig. 1B). At the initial selection, the mutants that were commonly started from one seed and grown in the same growing conditions for one year were washed and compared, which showed distinctive difference in numbers of root and tillers (Fig. 1). In the second year, WT and two mutants started from three tillers commonly exhibited significantly different performance. The higher tillering mutants showed thicker stem diameter and more leaf numbers, but shorter plant height in comparison with WT under flowering stage at the end of the experiment (Fig. 2). During the heading period in October, the tiller numbers for both mutants, CNU03BR22 and CNU03BR24 were 38.7 per pot, while WT (CNU03A01) produced 29.7, indicating 30\% higher to WT. Similarly stem diameters for two mutants CNU03BR22 and CNU03BR24 were 1.3 and 1.5 times higher, respectively when compared with WT. Also leaf numbers were 213 and 190 per pot for CNU03BR22 and CNU03BR24 mutants, respectively, while WT had 136 leaves. In contrast, plant height of WT was $135 \mathrm{~cm}$, while CNU03BR22 and CNU03BR24 mutants were $126 \mathrm{~cm}$ and $102 \mathrm{~cm}$, respectively.

Generally dwarfism along with higher tillers was reported to be associated with suppression of apical dominance controlled by auxin and more acropetal movement of cytokinin produced in root (McSteen, 2009). Another phytohormonestrigolactone was recently reported to suppress shoot branching (Gong et al., 2012). Two mutants showing higher tiller numbers in this study would be good resources to clarify factors such as genes, hormones, and any environmental conditions involving in axillary bud initiation and outgrowth, because CNU03BR22 remained to be taller as like WT, but CNU03BR24 was shorter. One of the targets will be a miR393 microRNA which downregulates auxin receptor and transporter genes such as OsTIR1, OsAFB2, and OsAux1 and promotes tillering and early flowering by repression of tillering inhibitor, TBI (Xia et al., 2012). Interestingly, two contrasting genes which include a reduced culm number $1(R c n l)$ gene promoting tillering or culm length and a dominant tillering dwarf (D3) gene reducing tiller number were reported to play independently, implying that plant stature is not always associated with tiller numbers as found in two mutants in this study (Yasuno et al., 2007).

Complemented with the above morphological traits which are more likely influenced by environmental factors, identification of mutants at DNA or cellular level will be a desirable strategy. A ploidy modification caused by nuclear DNA content was investigated in this study. However, no change in ploidy level was detected in two mutants when compared with diploid M. sinensis (Fig. 3A) and tetraploid M. sacchariflorus (Fig. 3B). Some morphological and physiological variations were reported to be associated with changes in DNA content, in which the ploidy level was not significantly different in 
banana when exposed to gamma radiation (Shadia et al., 2002). Due to increase in DNA content in banana mutants when exposed to low to higher doses of the gamma ray, more precise measurement of nuclear DNA content might be useful to identify mutants in Miscanthus. Additionally, target genes that regulate phenotypes of interest can be cloned and sequenced, resulting in verification of deletion or insertion of the allele affected by the ionizing mutagenesis.

Identification of accessions or ecotypes within a species or at the inter-specific level has been achievable at the molecular level since many types of molecular markers have been applicable (Chen et al., 2010; Kim et al., 2007). Among many reported DNA fingerprinting tools, the ITS region were found to be suitable due to its PCR efficiency and sequence divergence. Sequences of the ITS regions and intervening 5.8S region of two Miscanthus mutants exhibiting higher number of tillers were compared with those of WT (Table 1). Total length of three regions was $611 \mathrm{bp}$, which was consisted of ITS1, 5.8S, and ITS2 with 211, 164, and $227 \mathrm{bp}$, respectively (Fig. 4). All the accessions had the same size for ITS1, 5.8S, and ITS2 regions, but the sequences were different as seen in $\mathrm{G}+\mathrm{C}$ percentage, where the $\mathrm{G}+\mathrm{C}$ content of two mutants
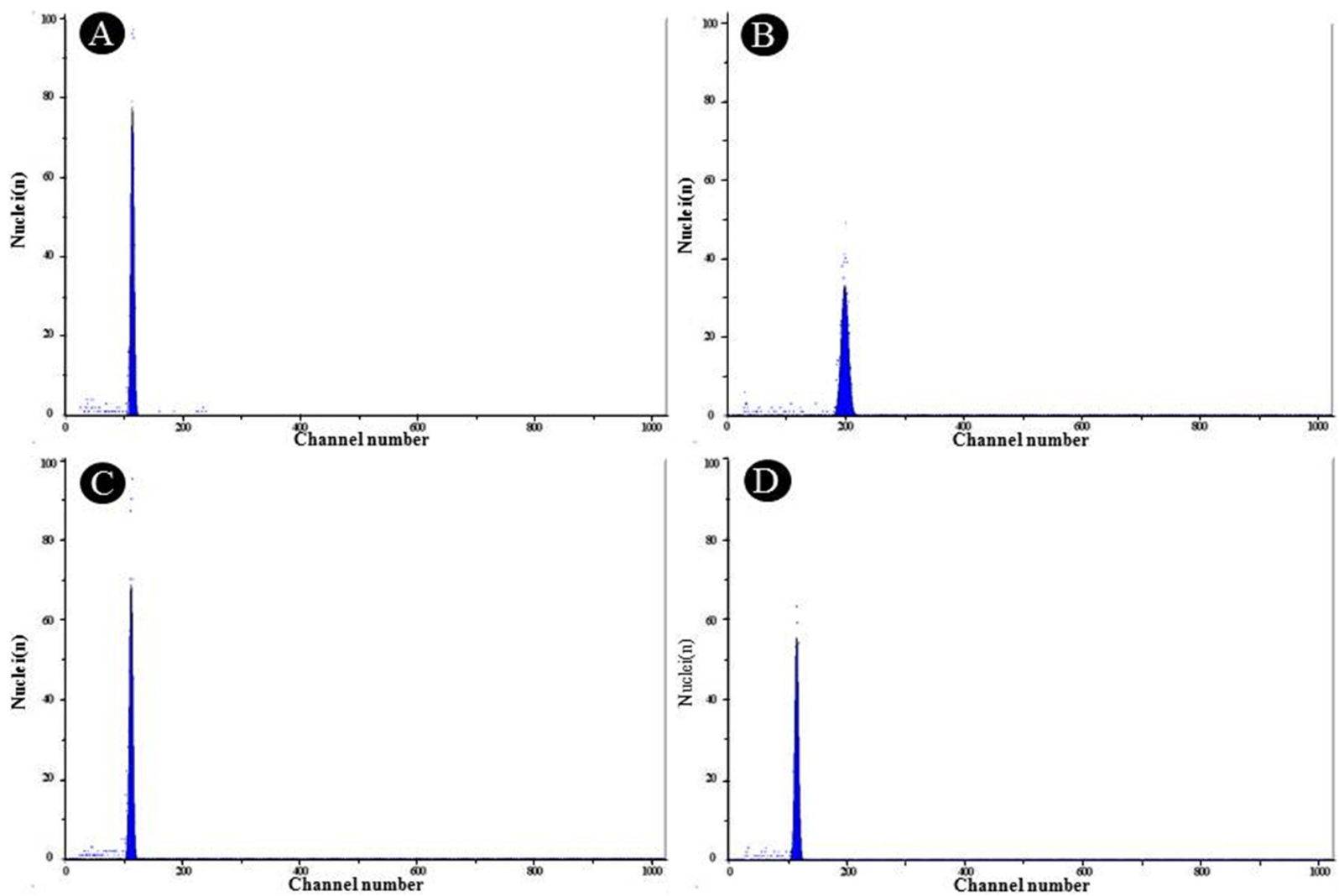

Fig. 3. Flow cytometric analysis of DAPI-stained DNA nuclei from diploid M. sinensis (A;WT), tetraploid M. sacchariflorus (B) and two mutants (C, CNU03BR22; D, CNU03BR24) which were induced by gamma ray treatment at 125 Gy.

Table 1. Sequence length and G+C content (\%) of ITS1, 5.8S and ITS2 regions of WT and the derived two mutants of Miscanthus

\begin{tabular}{|c|c|c|c|c|c|c|}
\hline \multirow{2}{*}{ Genotype } & \multicolumn{2}{|c|}{ ITS1 } & \multicolumn{2}{|c|}{$5.8 \mathrm{~S}$} & \multicolumn{2}{|c|}{ ITS2 } \\
\hline & Length (bp) & $\mathrm{G}+\mathrm{C}(\%)$ & Length (bp) & $\mathrm{G}+\mathrm{C}(\%)$ & Length (bp) & $\mathrm{G}+\mathrm{C}(\%)$ \\
\hline CNU03A01 (WT) & 211 & $135(63.9)$ & 164 & $93(57.0)$ & 226 & $152(67.2)$ \\
\hline CNU03BR22 & 211 & $134(63.5)$ & 164 & $93(57.0)$ & 226 & $154(68.1)$ \\
\hline CNU03BR24 & 211 & $133(63.0)$ & 164 & $93(57.0)$ & 226 & $153(67.7)$ \\
\hline
\end{tabular}


showed less in ITS1 and more in ITS2 (Table 1). There was no sequence change in $5.8 \mathrm{~S}$ subunit for three genotypes, while five bases were changed in both ITS1 and ITS2 of the mutants compared to WT. The base changes were occurred more in the 5' side of ITS1, while the event was relatively evenly spaced from 500 to 541 bp in ITS2 (Fig. 4). This result demonstrates that ITS regions can be used to identify the Miscanthus higher tillering mutants which were induced by a gamma radiation as suggested in other reports (Kress et al.,
2005; Chen et al., 2010).

One of the advantages using the ITS regions for mutant identification in this study is its bi-parental inheritability, which may distinguish those mutants from any artificial hybrids. Because limited genomic sequences of the genus Miscanthus are publicly available, the ITS regions were amplified by designing PCR primers of other zoysiagrass sequence (NCBI genebank no. HQ600481), indicating the potential application of the ITS sequences to other grasses for phylogentic divergence.
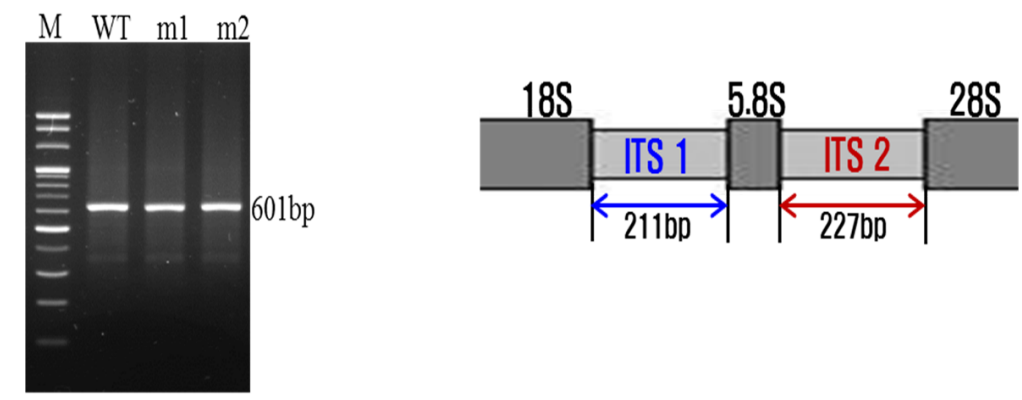

CNU03A01 CNU03BR22 CNUO3BR24

CNU03A01 CNU03BR22 CNU03BR24

CNU03A01 CNU03BR22 CNUO3BR24

CNU03A01 CNU03BR22 CNU03BR24

CNU03A01 CNU03BR22 CNUO3BR24

CNU03A01 CNU03BR22 CNU03BR24

CNU03A01 CNUO3BR22 CNU03BR24

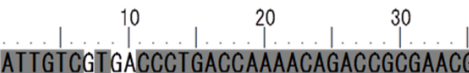

\section{ATTC}

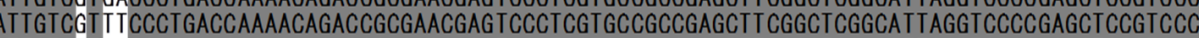
ATTGTCT TGACCCTGACCAAAACAGACCGCGAACGAGTCCCTCGTGCCGCCGAGCTTCGGCTCGGCATTAGGTCCCCGAGCTCCGTCCCG

\section{ITS1 TII}

$$
100 \quad 110,120
$$

GGG GGGCGTAGGGGCCACAACAGAACCCACGGCGCCTTAGGCGTCAAGGAACACTTATATTGCGTTGCTCGGCGGGGCGGTCGGCCTGCCTTC

\begin{tabular}{|l|l|l|l|l|l|l|l|}
\hline 190 & 200 & 210 & 220 & 230 & 240 & 250 & 260
\end{tabular}

CGCTCCCCGCGCAGCGAT GATATCTTAATCCACATGACTCTCGGCAACGGATATCTCGGCTCTCGCATCGATGAAGAACGTAGCAAAATG

CGCTCCCCGCGCAGCGATGATATCTTAATCCACATGACTCTCGGCAACGGATATCTCGGCTCTCGCATCGATGAAGAACGTAGCAAAATG CGCTCCCCGCGCAGCGATGATATCTTAATCCACATGACTCTCGGCAACGGATATCTCGGCTCTCGCATCGATGAAGAACGTAGCAAAATG
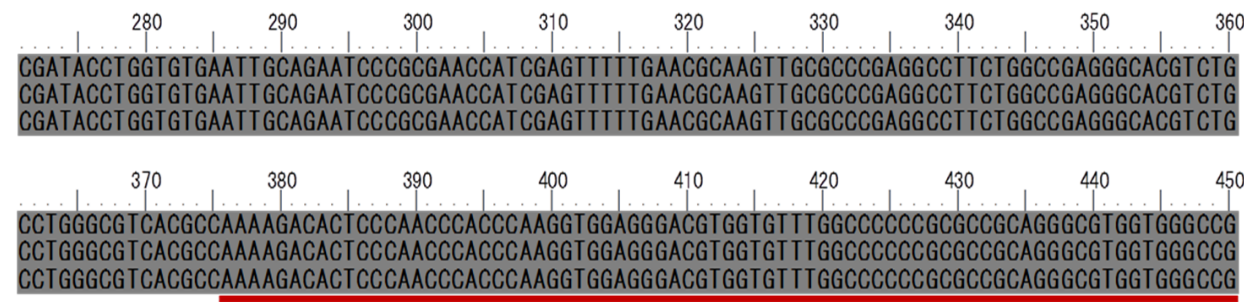

CCTGGGCGTCACGCCAAAAGACACTCCCAACCCACCCAAGGTGGAGGGACGTGGTGTTTGGCCCCCCGCGCCGCAGGGCGTGGTGGGCCG

$$
\text { ITS } 2_{470}
$$

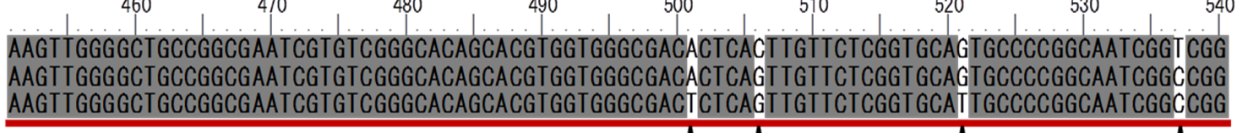
AAGTTGGGGCTGCCGGCGAATCGTGTCGGGCACAGCACGTGGTGGGCGACTCTCAGTTGTTCTCGGTGCATTGCCCCGGCAATCGGCCGG

$550 \quad 560 \quad 570 \quad 580 \quad 590 \quad 1600$

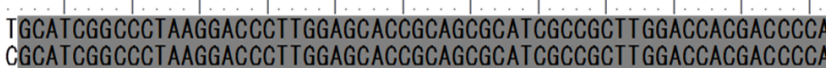

CGCATCGGCCCTAAGGACCCTTGGAGCACCGCAGCGCATCGCCGCTTGGACCACGACCCCA

$\Gamma$

Fig. 4. Successful PCR amplicons on $1 \%$ agarose gel (top left; $\mathrm{m}=100 \mathrm{bp}$ DNA marker, $\mathrm{wt}=$ wild type, $\mathrm{m} 1=\mathrm{CNU} 03 \mathrm{BR} 22, \mathrm{~m} 2=$ CNU03BR24), simplified structure and designated size (bp) of ITS regions (top right), and sequence variations (arrows) of both spacers linked to 5.8S region (bottom) of Miscanthus ribosomal DNA. 
Size of plant ITS regions ranges from 500 to $700 \mathrm{bp}$ long and the ribosomal gene repeats are abundant (hundreds to thousands) in plant genome, which facilitates the tool for convenient PCR amplification compared to other DNA barcode system (Kress et al., 2005; Chen et al., 2010).

Mutation breeding through diverse (physical, chemical, or biological) mutagens has been widely applied to various plant species. One limitation of the breeding method is to identify mutants, which mostly relies on phenotypic variations feasible to environmental extremes. As instrumental advance and genome sequences become more available and accessible, relatively efficient and precise identification tools such as STS (sequence tagged site) markers and universal ITS barcode can be good alternatives as shown in this study.

\section{Acknowledgement}

This work was supported by a grant from the Plant Molecular Breeding Center of the Next-Generation BioGreen 21Program, RDA (grant no. PJ0081312012).

\section{Literature Cited}

Beale, C.V. and S.P. Long. 1997. Seasonal dynamics of nutrient accumulation and partitioning in the perennial $\mathrm{C}_{4}$-grasses Miscanthus x giganteus and Spartina cynosuroides. Biomass Bioenergy 12:419-428.

Brosse, N., A. Dufour, X. Meng, Q. Sun and A. Ragauskas. 2012. Miscanthus: a fast-growing crop for biofuels and chemicals production. Biofuel. Bioprod. Bior. 6:580-598.

Chen, S., H. Yao, J. Han, C. Liu, J. Song, L. Shi, Y. Zhu, X. Ma, T. Gao, X. Pang, K. Luo, Y. Li, X. Li, X. Jia, Y. Lin and C. Leon. 2010. Validation of the ITS2 region as a novel DNA barcode for identifying medicinal plant species. PLoS One 5(1):e8613.

Dhanavel, D., S. Gnanamurthy and M. Girija. 2012. Effect of gamma rays on induced chromosomal variation in cowpea Vigna unguiculata (L.) Walp. Int. J. Curr. Sci. 245-250.

Eynon, R.T. 2011. Renewable energy consumption and electricity preliminary statistics 2010. http://www.eia.gov/ftproot/ renewables/pretrends10.pdf.

Gong, L., Y.J. Yang, and J. Zhou. 2012. Genes involved in the synthesis and signaling pathway of strigolactone, a shoot branching inhibitor. Biol. Plantarum 56:210-214

Greef, J.M. and M. Deuter. 1993. Syntaxonomy of Miscanthus x giganteus GREEF et DEU. Angew. Bot. 67:87-90.

Heaton, E.A., J. Clifton-Brown, T.B. Voigt, M.B. Jones and S.P. Long. 2003. Miscanthus for renewable energy generation: European Union experience and projections for Illinois. Mitigation and Adaptation Strategies for Global Change 9:433-451.

Henry, R.J. 2010. Evaluation of plant biomass resources available for replacement of fossil oil. Plant Biotechnol. J. 8:288-293.

Hodkinson, T.R., M.W. Chase, S.A. Renvoize. 2002. Characterization of a genetic resource collection for Miscanthus (Saccharinae, Andropogonea, Poaceae) using AFLP and ISSR PCR. Ann. Bot. 89:627-636.

Jones, M.B. and M. Walsh. 2001. Miscanthus: For Energy and Fibre, James \& James Ltd., London.

Kim, O.T., K.H. Bang, D.S. In, J.W. Lee, Y.C. Kim, Y.S. Shin, D.Y. Hyun, S.S. Lee, S.W. Cha and N.S. Seong. 2007. Molecular authentication of ginseng cultivars by comparison of internal transcribed spacer and 5.8S rDNA sequences. Plant Biotechnol. Rep. 1:163-167.

Kress, W.J., K.J. Wurdack, E.A. Zimmer, L.A. Weigt and D.H. Janzen. 2005. Use of DNA barcodes to identify flowering plants. P. Natl. Acad. Sci.(PNAS) 102:8369-8374.

Lewandowski, I., J.C. Clifton-Brown, J.M.O. Surlock and S. Huisman. 2000. Miscanthus: European experience with a novel energy crop. Biomass Bioenergy 19:209-227.

Li, X., M. Lassner and Y. Zhang. 2002. Deleteagene: a fast neutron deletion mutagenesis-based gene knockout system for plants. Comp. Funct. Genom. 3:158-160.

McSteen, P. 2009. Hormonal regulation of branching in grasses. Plant Physiol. 149:46-55.

Pyter, R.J., F.G. Dohleman and T.B. Voigt. 2010. Effects of rhizome size, depth of planting and cold storage on Miscanthus $\mathrm{x}$ giganteus establishment in the Midwestern USA. Biomass Bioenergy 34:1466-1470.

Rayburn, A.L., J. Crawford, C.M. Rayburn and J.A. Juvik. 2009. Genome size of three Miscanthus species. Plant Mol. Biol. Rep. 27:184-188.

Shadia, A.R., R.Y. Othman and M. Chai. 2002. Plojdy analysis and DNA content of mutant banana "Pisang Berangan" using flow cytometry. Biotropia 19:47-56.

Sorensen, A., P.J. Teller, T. Hilstrom and B.K. Ahring. 2008. Hydrolysis of Miscanthus for bioenthanol production using 
dilute acid presoaking combined with wet explosion pretreatment and enzymatic treatment. Bioresource Technol. 99:6602-6607.

Swaminathan, K., W.B. Chae, T. Mitros, K. Varala, L. Xie, A. Barling, K. Glowacka, M. Hall, S. Jezowski, R. Ming, M. Hudson, J.A. Juvik, D.S. Rokhsar and S.P. Moose. 2012. A framework genetic map for Miscanthus sinensis from RNAseqbased markers shows recent tetraploidy. BMC Genomics 13:142.

Tindall, K.R., J. Stein and F. Hutchinson. 1988. Changes in DNA base sequence induced by gamma-ray mutagenesis of lambda phage and prophage. Genetics 118:551-560.

van Harten, A.M. 1998. Mutation Breeding: Theory and
Practical Applications, Cambridge University Press, London. Venendaal, R., U. Jorgensen and C. Foster. 1997. European energy crops: a synthesis. Biomass Bioenergy 13:147-185.

Xia, K., R. Wang, X. Ou, Z. Fang, C. Tian, J. Duan, Y. Wang and M. Zhang. 2012. OsTIR1 and OsAFB2 downregulation via OsmiR393 overexpression leads to more tillers, early flowering and less tolerance to salt and drought in rice. PLoS One 7(1):e30039.

Yasuno, N., Y. Yasui, I. Takamure and K. Kato. 2007. Genetic interaction between 2 tillering genes, reduced culm number 1 ( $r c n 1)$ and tillering dwarf gene $d 3$, in rice. J. Hered. 98:169-172.

(Received 2 November 2012 ; Revised 23 November 2012 ; Accepted 3 December 2012) 